\title{
GLÓQUIDAS DEL FRUTO DE Opuntia albicarpa SCHEINVAR Y SU HIDRÓLISIS PARA USO POTENCIAL AGRONÓMICO
}

\section{GLOCHIDS FROM THE FRUIT OF Opuntia albicarpa SCHEINVAR AND THEIR HYDROLYSIS FOR POTENTIAL AGRONOMIC USE}

\author{
Alonso Ulloa-Leitón' ${ }^{1}$ M. Edna Álvarez-Sánchez*, Cecilia García- \\ Osorio ${ }^{2}$, Francisco Gavi-Reyes ${ }^{3+}$ y Ranferi Maldonado-Torres ${ }^{1}$
}

\begin{abstract}
'Universidad Autónoma Chapingo, Departamento de Suelos, Chapingo, Estado de México, México. ${ }^{2}$ Colegio de Postgraduados (CP), Posgrado en Recursos Genéticos y Productividad-Fruticultura, Montecillo, Estado de México, México. ${ }^{3} \mathrm{CP}$, Posgrado en Hidrociencias, Montecillo, Estado de México, México.
\end{abstract}

*Autor de correspondencia (edna_alvarez30@yahoo.com.mx)

\section{RESUMEN}

Las glóquidas de la tuna constituyen un residuo orgánico que se deriva del beneficiado del fruto, previo a su comercialización. Este residuo se desecha en bolsas plásticas a la orilla de plantaciones 0 en basureros no regulados donde se acumula debido a su alta resistencia a la degradación natural y a la combustión. El objetivo de la presente investigación fue estudiar la estructura externa, composición química, rendimiento de hidrólisis y contenido de nutrimentos vegetales a partir de la hidrólisis de las glóquidas de tuna. La estructura externa se observó por microscopio electrónico de barrido; se encontró que las glóquidas poseen barbas retrorsas. Las dimensiones de las glóquidas en sus planos transversal y longitudinal fueron de $67.3 \pm 9.56$ y $1667 \pm 292 \mu \mathrm{m}$, respectivamente. Los análisis químicos indicaron que las glóquidas se componen de $41.15 \%$ celulosa, $41.20 \%$ hemicelulosa y 5.27 $\%$ lignina; presentan una densidad de $0.157 \mathrm{~g} \mathrm{~mL}^{-1}$. Los valores mayores de degradación, azúcares reductores y grados Brix se obtuvieron a partir de la hidrólisis con ácido sulfúrico, hidróxido de potasio y ácido fosfórico. El contenido de nutrimentos vegetales de las glóquidas hidrolizadas se comparó con el reportado en soluciones nutritivas comerciales y se valoró que tienen gran potencial como fertilizante foliar en cultivos agrícolas.

Palabras clave: Azúcares reductores totales, glóquidas, grados Brix, hidrólisis ácida y alcalina.

\section{SUMMARY}

Glochids of prickly pear constitute an organic residue derived from the processing of the fruit prior to its commercialization. This residue is disposed into plastic bags at the edge of plantations or in unregulated landfills where it accumulates due to its high resistance to natural degradation and combustion. The objective of this investigation was to study the external structure, chemical composition, hydrolysis yield and content of plant nutrients after hydrolysis of prickly pear glochids. The external structure was observed through a scanning electron microscope; it was found that they have retrorse barbs. The dimensions of glochids in their transverse and longitudinal planes were $67.3 \pm 9.56$ and $1667 \pm 292 \mu \mathrm{m}$, respectively. Chemical analyses indicated that glochids are composed of $41.15 \%$ cellulose, $41.20 \%$ hemicellulose and $5.27 \%$ lignin; they have a density of $0.157 \mathrm{~g} \mathrm{~mL}^{-1}$. The highest degradation values, reducing sugars and Brix degrees were obtained from hydrolysis with sulfuric acid, potassium hydroxide and phosphoric acid. The content of plant nutrients in hydrolyzed glochids was compared to that reported in commercial nutrient solutions and it was valued as having great potential as foliar fertilizer in agricultural crops.
Index words: Acid and alkaline hydrolysis, Brix degrees, glochids, reducing sugars.

\section{INTRODUCCIÓN}

La producción de tuna representa una fuente importante de ingresos para los productores de las regiones semiáridas de México, ya que por cada unidad monetaria invertida en la producción de tuna se obtienen 3.16 de ganancia (CallejasJuárez et al., 2009). Desafortunadamente, la producción de la tuna genera impactos negativos sobre el ambiente. Después de la cosecha, los frutos son procesados para eliminar las glóquidas, popularmente conocidas como espinas o "ahuates".

Este subproducto es un problema para el productor, ya que no le conocen utilidad; además, tiene alta resistencia a la degradación biológica y a la quema, por lo que son vertidas a orillas de las plantaciones o en los basureros sin ningún control sanitario. Generalmente, las glóquidas son envasadas en bolsas de plástico, las cuales, al tratarse de un material no degradable, también se suman al efecto negativo en el ambiente. Se estima que en las 2532 ha dedicadas a la producción de tuna en el municipio de Nopaltepec, Estado de México, en el período de 2010 a 2015 se produjeron 80.1 t de glóquidas, las cuales fueron desechadas en 5337 bolsas plásticas (Ulloa, 2020; Com. Pers. $)^{1}$.

El problema ambiental de las glóquidas no sólo se relaciona con las bolsas de plástico que se usan para su disposición, también representa un riesgo para la salud de las personas, ya que pueden incrustarse en la piel causando diferente grado de irritación (Doctoroff et al., 2000) o

UUlloa L. A. (2020) Manejo integral de las glóquidas del nopal tunero. Tesis de Maestría en Ciencias en Agroforestería para el Desarrollo Sostenible. Universidad Autónoma Chapingo Chapingo, Estado de México. 71 p 
lastimar los ojos (Mustafa-Odat et al., 2014). La relevancia del peligro de salud que estas estructuras representan ha motivado el estudio de las heridas que provocan (Dieter et al., 2017) y la documentación de los métodos para su eliminación de la piel cuando no están enterradas (Ford et al., 2019), antes de llegar a procedimientos quirúrgicos. También existe información para los casos donde ya no están visibles para su extracción, así como de los procedimientos de detección previa (Yousef et al., 2017).

La dificultad a la degradación de las glóquidas se relaciona con la dureza de su estructura, asociada con su grado de cristalización (20-60 \%) y al alto grado de alineación (1-2.50) de las fibras de celulosa, lo cual les confieren resistencia similar o superior a materiales sintéticos como la fibra de vidrio (Martinez et al., 2017; Com. Pers.) $)^{2}$. Gindl-Altmutter y Keckes (2012) compararon la estructura y las propiedades mecánicas de las espinas de los cladodios de Opuntia ficus-indica con la madera de abeto, y encontraron que la mayor resistencia de las espinas se debe a que su pared celular se compone únicamente de arabinosa (50 \%) y celulosa (50 \%), y a la alta alineación y cristalización de las fibras de este polisacárido, en comparación con la pared de las células de la madera que tiene $50 \%$ de celulosa, $25 \%$ de lignina y $25 \%$ de polisacáridos no estructurales.

Existen reportes sobre la composición química del nopal para cladodios, tuna, jugo de tuna y semillas, incluyendo macro y micronutrientes (Chahdoura et al., 2015; Mustafa-Odat et al., 2014), así como minerales (Kalegowda et al., 2015); sin embargo, para las glóquidas del fruto no se han realizado estudios para conferirles algún uso alternativo. En la búsqueda de posibles usos agrícolas de estas estructuras, el conocimiento sobre su composición y estructura, así como su contenido mineral son determinantes.

Con base en lo anterior, se planteó como objetivo estudiar la estructura externa, composición química, rendimiento de hidrólisis y contenido de nutrimentos vegetales a partir de la hidrólisis ácida y alcalina de las glóquidas de tuna.

\section{MATERIALES Y MÉTODOS}

\section{Colecta de glóquidas}

El área de muestreo fue la zona tunera de Nopaltepec,

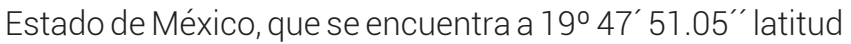
Ny $98^{\circ} 40^{\prime} 47.95^{\prime \prime}$ longitud O. En esta zona se recolectaron $10 \mathrm{~kg}$ de glóquidas en los talleres donde los ejidatarios de

${ }^{2}$ Martinez J., T. Stewart and P. Szeto (2017) The relationship between structural parameters and mechanical properties of cactus spines. Senior Project. California Polytechnic State University. San Luis Obispo, California, USA. 106 p. la comunidad de San Felipe Teotitlán benefician la tuna.

\section{Estructura externa de las glóquidas, análisis proximal y nutrimental}

De la muestra se campo se tomaron $200 \mathrm{~g}$ de glóquidas y se lavaron con agua destilada para eliminar residuos orgánicos y polvo. Estructuras fueron colocadas durante 5 min en un equipo Sputter Quorum Q150 ES (Quorum Technologies Ltd., East Sussex, Inglaterra) para cubrirlas con oro y ser observadas a diferentes magnificaciones en el microscopio electrónico de barrido (MEB) Modelo JSM-6390 (Hitachi High-Tech America, Inc., Pleasanton, California, EUA). El aspecto externo del ápice y corte transversal de la glóquida se observó con el MEB de la Unidad de Microscopía Electrónica (UME) del Colegio de Postgraduados, en tanto que el aspecto de la estructura externa de glóquidas molidas y trozadas, así como la determinación de las dimensiones (largo y diámetro, en 13 muestras) con el MEB del Laboratorio Nacional de Investigación y Servicios Agroalimentarios y Forestales (LANISAF) de la Universidad Autónoma Chapingo. Adicionalmente, se midió la densidad de los residuos con el método de la probeta (peso/volumen), en seis muestras.

\section{Determinación de celulosa, hemicelulosa y lignina}

Del material limpio se tomó una muestra de aproximadamente $20 \mathrm{~g}$ y se secó en estufa de aire forzado a $40{ }^{\circ} \mathrm{C}$ por tres días. Una vez seca, se molió hasta pasar por malla 40. La determinación se realizó en muestras de $0.5 \mathrm{~g}$ con tres repeticiones conforme al método de Van Soest et al. (1991).

\section{Determinación del contenido nutrimental de las glóquidas}

El contenido de macronutrimentos (, , P, K, Ca y Mg) y micronutrimentos (Fe, Cu, Zn, Mn, B y Si) se determinó mediante digestión ácida de las glóquidas con ácido nítrico:ácido perclórico (4:2, v:v). La cuantificación de Ca, $\mathrm{Mg}, \mathrm{Fe}, \mathrm{Mn}, \mathrm{Cu}, \mathrm{Zn}$ y Si se realizó por espectrofotometría de absorción atómica y K por flamometría; B por Azometina-H, $S$ por turbidimetría y $P$ en el complejo vanadomolíbdico en espectrofotómetro de luz visible. El N se determinó por el método semi-micro Kjeldahl (Alcántar y Sandoval, 1999). Estos análisis se realizaron por triplicado.

\section{Hidrólisis de las glóquidas}

En este proceso se usaron muestras de glóquidas secas, limpias y tamizadas. Los tratamientos de hidrólisis (Cuadro 1) consistieron en el uso de soluciones ácidas (a partir de ácido sulfúrico y fosfórico), agua oxigenada y soluciones 
alcalinas (hidróxido de potasio, hidróxido de sodio y de calcio) en diferentes relaciones (peso de muestra/volumen de solución) y condiciones de pre-digestión, como se indica en el Cuadro 1. Cada tratamiento tuvo cuatro repeticiones.

La solución con sólidos residuales producto de la hidrólisis fue colada en papel de rápida filtración. Las soluciones resultantes se neutralizaron a $\mathrm{pH}$ de $7 \mathrm{con}$ ácido nítrico $30 \%$ o hidróxido de potasio $20 \%$, con la finalidad de estabilizar los azúcares reductores. En cada caso, los sólidos retenidos en el papel, junto con éste, se secaron a $45{ }^{\circ} \mathrm{C}$ durante $12 \mathrm{~h}$ y finalmente se determinó el peso seco de dichos sólidos en una balanza digital Ohaus (Modelo 30208447, Parsippany-Troy Hills, New Jersey, EUA). La valoración de los métodos de hidrólisis se basó en el rendimiento de azúcares reductores totales disueltos, grados Brix ( $\left.{ }^{\circ} \mathrm{Bx}\right), \mathrm{pH}$, conductividad eléctrica de la solución, así como el porcentaje de degradación de las glóquidas (con base en los sólidos residuales). Además, se determinó el contenido nutrimental de $\mathrm{P}, \mathrm{K}, \mathrm{Ca}, \mathrm{Mg}, \mathrm{Fe}, \mathrm{Mn}$, $\mathrm{Cu}, \mathrm{Zn}$ y $\mathrm{B}$ directamente en el extracto producto de tres tratamientos de hidrólisis, siguiendo los procedimientos indicados en el apartado anterior. La selección del tipo de hidrólisis para conocer la composición mineral del extracto se basó en los rendimientos más altos de degradación, azúcares reductores $\mathrm{y}^{\circ} \mathrm{Bx}$.

\section{Determinación de los azúcares reductores totales}

Este análisis se hizo en la solución resultante de la hidrólisis de acuerdo con la técnica DNS (Gómez, 2008;
Com. Pers.) $)^{3}$, usando glucosa como estándar. Para obtener la curva de calibración se realizaron pruebas preliminares para precisar la amplitud lineal de la curva y cumplir con la Ley de Lambert y Beer, por lo que se partió de una solución cuya concentración de glucosa fue de $585 \mathrm{\mu g} \mathrm{mL}^{-1}$, que se diluyó a diferentes volúmenes para obtener diferentes concentraciones (Cuadro 2).

La preparación de $100 \mathrm{~mL}$ de la disolución DNS consistió en disolver por separado cada uno de los reactivos en el siguiente orden y con agitación constante: hidróxido de sodio $(1.4 \mathrm{~g})$, fenol $(0.558 \mathrm{~g})$, metasulfito $(0.49 \mathrm{~g})$, tartrato de sodio y potasio $(2.9 \mathrm{~g})$ y ácido $3-5$ dinitrosalicílico (1.0 g) y se agitó durante 30 min adicionales; posteriormente, la disolución se aforó a $100 \mathrm{~mL}$ y se pasó a un frasco de vidrio color ámbar cubierto con papel aluminio y se refrigeró a $4^{\circ} \mathrm{C}$.

Se tomaron $1000 \mu \mathrm{L}$ de cada punto de la curva, se adicionaron $1000 \mu \mathrm{L}$ de la disolución DNS y se colocaron en baño María durante $5 \mathrm{~min}$; posteriormente, se colocaron en un baño frío para detener la reacción. Se leyó la absorbancia en un espectrofotómetro Genesys 10 UV (Thermo Fisher Scientific, Waltham, Massachusetts, EUA) a $540 \mathrm{~nm}$. Para los extractos de las muestras se siguió el mismo procedimiento. Las determinaciones se realizaron por cuadruplicado.

${ }^{3}$ Gómez T. F. (2008) Métodos secuenciales de pretratamiento químico y enzimático de residuos agrícolas para la producción de metano. Tesis de Maestría. Instituto Potosino de Investigación Científica y Tecnológica. San Luis Potosí, México. 78 p.

Cuadro 1. Tratamientos de hidrólisis de las glóquidas de tuna.

\begin{tabular}{|c|c|c|c|c|c|c|}
\hline $\begin{array}{l}\text { Tratamiento } \\
\text { No. }\end{array}$ & $\begin{array}{c}\text { Solución } \\
\text { (concentración en \%) }\end{array}$ & $\begin{array}{l}\text { Relación } \\
\text { (g material:mL } \\
\text { disolución) }\end{array}$ & $\begin{array}{l}\text { Predigestión } \\
\text { (h) }\end{array}$ & $\begin{array}{c}\text { Baño } \\
\text { María }\left({ }^{\circ} \mathrm{C}\right)\end{array}$ & $\begin{array}{l}\text { Agitación } \\
\text { (h) }\end{array}$ & $\begin{array}{c}\text { Neutralización } \\
\text { con ácido o base } \\
\text { (concentración en \%) }\end{array}$ \\
\hline 1.1 & $\mathrm{H}_{2} \mathrm{SO}_{4} \quad$ (15) & $1: 30(2 / 60)$ & 24 & 60 & 3 & $\mathrm{KOH}(40)$ \\
\hline 1.2 & & & & $\sin$ & & $\mathrm{KOH}(50)$ \\
\hline 2.1 & $\mathrm{H}_{3} \mathrm{PO}_{4}$ & 1:30 (2:60) & 24 & 60 & 3 & $\mathrm{KOH}(40)$ \\
\hline 2.2 & & & & $\sin$ & & $\mathrm{KOH}(50)$ \\
\hline 3.1 & $\mathrm{NaOH} \quad$ (15) & 1:30 (2:60) & 24 & 60 & 3 & $\mathrm{HNO}_{3}(50)$ \\
\hline 3.2 & & & & $\sin$ & & \\
\hline 4 & $\mathrm{H}_{2} \mathrm{O}_{2} \quad(10)$ & 1:20 (3:60) & $\mathrm{n} / \mathrm{a}^{+}$ & $\sin$ & 6 & $\mathrm{KOH}(20)$ \\
\hline 5.1 & $\mathrm{Ca}(\mathrm{OH})_{2}(0.2)$ & 1:25 (2:50) & $\mathrm{n} / \mathrm{a}$ & 60 & 6 & $\mathrm{HNO}_{3}(30)$ \\
\hline 5.2 & & & & $\sin$ & & \\
\hline 6.1 & $\mathrm{PHP}^{+}$ & 1:10 (3:30) & 24 & 40 & 3 & $\mathrm{KOH}(20)$ \\
\hline 6.2 & & & & $\sin$ & & \\
\hline 7.1 & $\mathrm{KOH}$ & $1: 30(2: 60)$ & 24 & 60 & 3 & $\mathrm{HNO}_{3}(30)$ \\
\hline 7.2 & & & & $\sin$ & & \\
\hline 8 & $\mathrm{Ca}(\mathrm{OH})_{2}(10)$ & 1:25 (2:50) & $\mathrm{n} / \mathrm{a}$ & 60 & 6 & $\mathrm{HNO}_{3}(30)$ \\
\hline
\end{tabular}

${ }^{\dagger}$ La neutralización a pH 7 se consiguió con $\mathrm{KOH} 20 \%$ aplicando $12.2 \mathrm{~mL}$ para el tratamiento 6.1 y $16.1 \mathrm{~mL}$ para el tratamiento 6.2 . 
Cuadro 2. Curva de calibración para la determinación de azúcares reductores.

\begin{tabular}{lcccc}
\hline Muestra & $\begin{array}{c}\text { Concentración de } \\
\text { glucosa }\left(\mu \mathrm{gL} \mathrm{m}^{-1}\right)\end{array}$ & Solución $(\mathrm{mL})$ & Volumen $\mathrm{H}_{2} \mathrm{O}(\mathrm{mL})$ & Volumen total $(\mathrm{mL})$ \\
\hline Blanco & 0.0 & 0.0 & 1.0 & 1.0 \\
1 & 58.5 & 0.1 & 0.9 & 1.0 \\
2 & 175.5 & 0.3 & 0.7 & 1.0 \\
3 & 292.5 & 0.5 & 0.5 & 1.0 \\
4 & 409.5 & 0.7 & 0.3 & 1.0 \\
5 & 526.5 & 0.9 & 0.1 & 1.0 \\
6 & 585.0 & 1.0 & 0.0 & 1.0 \\
\hline
\end{tabular}

\section{Análisis estadístico}

A los valores de longitud, diámetro y densidad, contenidos de celulosa, hemicelulosa y lignina de glóquidas, así como a los contenidos de minerales en la solución resultante de la hidrólisis de dichas estructuras se les determinó su desviación estándar ( $\mathrm{Sd}$ ) con base en el número de repeticiones de las mediciones realizadas; por ello, en los promedios de dichas variables se acompañan del valor 土 Sd. Las variables rendimiento de la hidrólisis, azúcares reductores, grados Brix y conductividad eléctrica fueron sometidos a análisis de varianza y prueba de medias (Tukey, $P \leq 0.05)$. Los estadísticos que soportan los resultados se obtuvieron con el programa INFOSTAT versión 2019.

\section{RESULTADOS Y DISCUSIÓN}

\section{Estructura externa de las glóquidas de tuna}

Las observaciones en microscopía electrónica de barrido mostraron que las glóquidas, de origen meristemático, tienen barbas retrorsas (dientes microscópicos) a lo largo de toda su estructura (Figura 1a), lo que facilita su adhesión a las superficies porosas. La longitud de las glóquidas fue de $1667 \pm 292 \mu \mathrm{m}$, con diámetro de $67.3 \pm 9.56 \mu \mathrm{m}$ (Figura 1b); la densidad de estas estructuras se estimó en $0.157 \pm$ $0.02 \mathrm{~g} \mathrm{~mL}^{-1}$.

La dureza de estas estructuras, entre otras características de su composición, parece estar asociada a las paredes celulares que son muy gruesas (Malainine et al., 2003; Martinez et al., 2017, Com. Pers. ${ }^{2}$ ), y en consecuencia, un lumen muy reducido (Figura 1b); además, se apreció que las glóquidas después de ser molidas y tamizadas no se pulverizaron, sólo quedaron trozadas (Figura 2), pero disminuyó su adherencia a la piel.

La estructura externa de los glóquidas del presente estudio es similar a la de otras especies de Opuntia, conforme a lo reportado en la literatura (Scheinvar et al.,
2015). El conocimiento de su estructura y composición es de vital importancia, ya que de ésta depende la facilidad o complejidad para que una sustancia pueda atacarla y lograr extraer los elementos minerales de interés; ésta es la primera resistencia que se debe vencer para su extracción.

En los espacios de las barbas retrorsas (Figura 1a) se encontraron esporas de hongos que germinaron y emergieron cuando estas estructuras se usaron como sustrato para la geminación de semillas. Estas observaciones desarrollaron la idea de utilizarlas como sustrato para la producción de hongos comestibles (investigación en proceso); al parecer, el espacio entre las barbas y la conformación de las fibras favorece la retención de agua, pues actúa como microporo y a su vez permite la aireación, lo cual no sucedería si la superficie fuera lisa, como ocurre con las glóquidas de otras especies de Opuntia (Martínez-González et al., 2015) o incluso, la espina de la misma Opuntia ficus-indica (Malainine et al., 2003; Cooper et al., 2013, Com. Pers. ${ }^{4}$ ). Al efectuar los análisis de la presente investigación se observó que las glóquidas en contacto con el agua desprenden taninos que tiñen el agua de color rojo, lo cual podría ser otra línea de investigación sobre los usos potenciales de dichas estructuras del fruto de Opuntia.

\section{Contenido de celulosa, hemicelulosa y lignina}

La composición de la pared de las células de las glóquidas mostró proporciones altas tanto de celulosa (41.15 $\pm 0.2 \%)$ como de hemicelulosa (41.2 $\pm 0.2 \%$ ) y sólo $5.27 \pm 0.2 \%$ de lignina. Es conocido que la celulosa es el polímero estructural de las células que controla las propiedades mecánicas de las plantas superiores. Este polímero en la pared celular se encuentra en forma de microfibrillas organizadas en fibras, cuyas cadenas de celulosa están alineadas paralelas al eje microfibril. Esta organización confiere a las microfibrillas

\footnotetext{
${ }^{4}$ Cooper M., J. Goldstein and T. Tarlton (2013) Strength testing and material characterization of cactus spines. Senior Project. California Polytechnic State University. San Luis Obispo, California, USA. $26 \mathrm{p}$.
} 

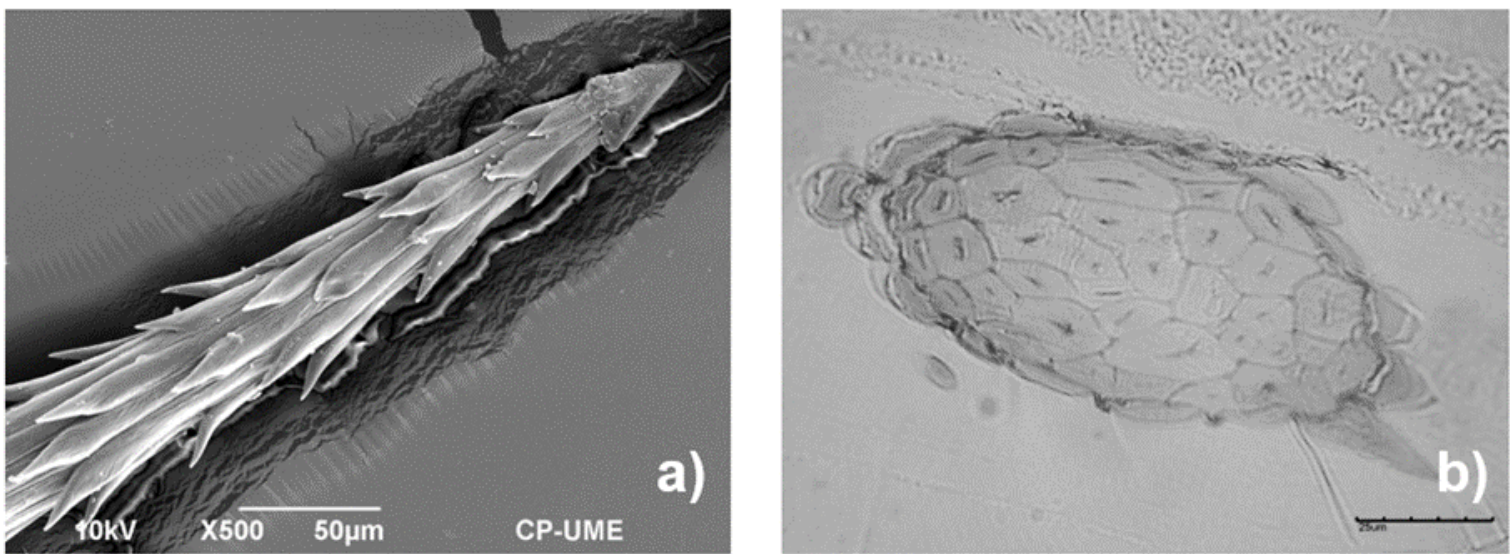

Figura 1. Aspecto externo de ápice (a) y corte transversal (b) de una glóquida de Opuntia albicarpa Scheinvar observados con MEB.
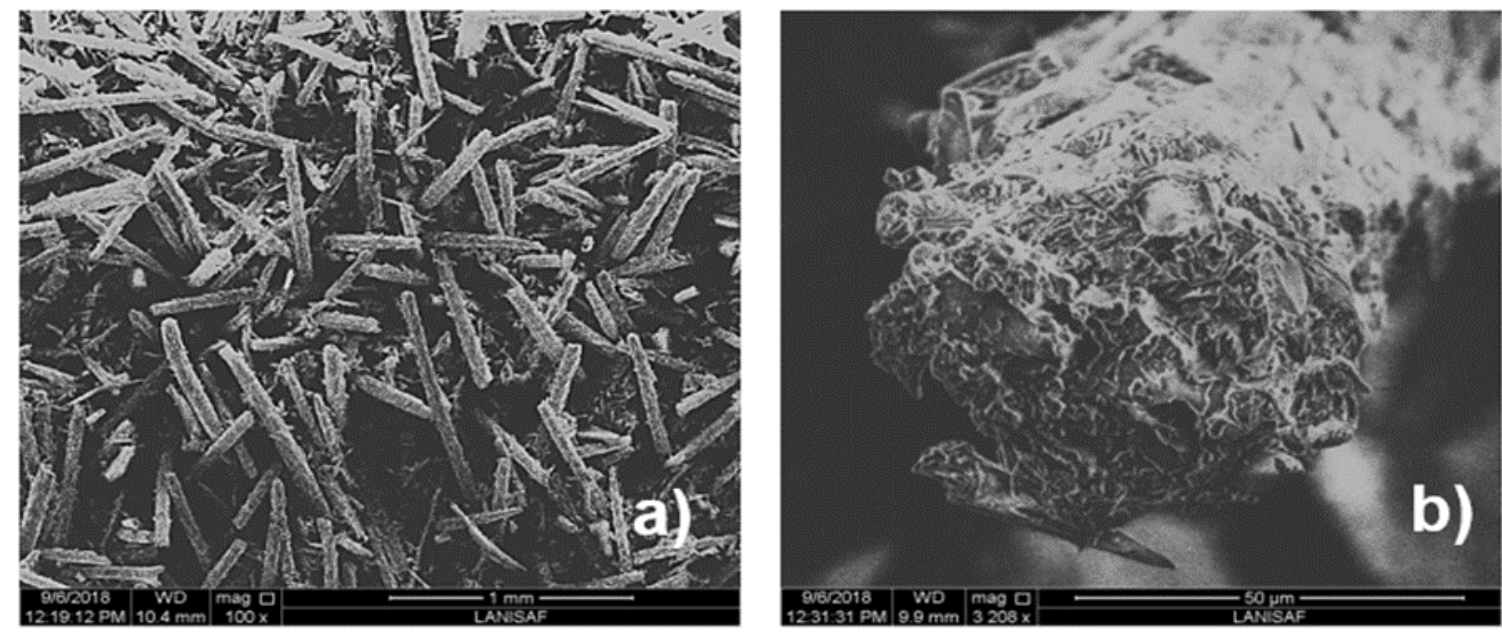

Figura 2. Estructura externa de glóquidas molidas 100x (a) y glóquida trozada 3208x (b) de Opuntia albicarpa Scheinvar observada con MEB.

propiedades de alta resistencia mecánica. Malainine et al. (2003) encontraron que la espina del cladodio de Opuntia ficus-indica contiene proporciones similares de celulosa/ hemicelulosa (51.2/47.5) y un bajo contenido de lignina (3.6 $\%)$. El sistema de celulosa/hemicelulosa se encuentra en forma cristalina y las fibrillas se disponen en forma paralela, casi perfecta, al eje espinal; las microfibrillas de celulosa están inmersas en la matriz de hemicelulosa interactuando a nivel molecular (Gindl-Altmutter y Keckes, 2012; Vignon et al., 2004). Esta organización podría ser la responsable de la alta resistencia mecánica de estas estructuras, propiedades que podrían explicar la dureza de las glóquidas y la dificultad que podría resultar para su hidrólisis ácida o alcalina para el aprovechamiento de las moléculas de interés.

\section{Rendimiento de la hidrólisis}

El rendimiento de los procesos de hidrólisis, cuantificado como porcentaje de degradación de las glóquidas, así como el contenido de azúcares reductores totales, grados Brix $\left({ }^{\circ} \mathrm{Bx}\right)$ y conductividad eléctrica (CE) de las soluciones resultantes de dicha hidrólisis se presentan en el Cuadro 3.

Los mayores porcentajes de degradación y contenido de azúcares reductores se consiguieron con el tratamiento a base de ácido sulfúrico; asimismo, se observó que los valores de ambos indicadores fueron mayores con el pretratamiento de baño María, pero éste no afectó los ${ }^{\circ} \mathrm{Bx}$ ni la CE. Esto es un indicio de cómo pueden ser tratadas estas estructuras para acelerar su degradación y aprovechar potencialmente algunos de sus componentes. La CE que se genera con este tipo de hidrólisis tampoco 
representa una limitante para el uso del extracto con fines agronómicos, por ejemplo en fertilización foliar.

El segundo y tercer mayor porcentaje de degradación de las glóquidas y azúcares reductores se obtuvieron con el tratamiento a base de hidróxido de sodio y ácido fosfórico. En ambos tratamientos el baño María no afectó a los parámetros evaluados ( ${ }^{\circ} \mathrm{Bx}$, azúcares reductores y CE). Es importante destacar que el pretratamiento de baño María ayudó a la degradación, pero no a la liberación de azúcares reductores. Finalmente, la hidrólisis con hidróxido de potasio fue el tercer mejor tratamiento en cuanto a porcentaje de degradación, contenido de azúcares reductores $\mathrm{y}^{\circ} \mathrm{Bx}$, pero el porcentaje de degradación promedio fue muy bajo $(<15$ \% con y sin baño María).

De acuerdo con algunos investigadores, la hidrólisis ácida con $\mathrm{H}_{2} \mathrm{SO}_{4} \circ \mathrm{HCl}$ en concentraciones menores al 5 $\%$ de distintas fuentes vegetales, proveen una adecuada hidrólisis de hemicelulosa con poca descomposición de azúcares reductores (Aguilar et al., 2008; Monsalve et al., 2006; Naranjo et al., 2016); sin embargo, la cristalinidad es una barrera física que dificulta la accesibilidad a los enlaces 1,4 glucosídicos que unen las cadenas de glucosa en la celulosa, razón por la cual concentraciones del 10 al 16 \% (Domínguez-Domínguez et al., 2012; Reis et al., 2007) han dado resultados prometedores en la hidrólisis de bagazo de caña de azúcar y bagazo de malta (Mussatto et al., 2006) para el aprovechamiento de etanol. Por otra parte, el tratamiento de hidrólisis con $\mathrm{H}_{3} \mathrm{PO}_{4}$ favorece la disolución de lignina con poco efecto sobre la celulosa (Domínguez-Dominguez et al., 2012), lo que explicaría la baja proporción de azúcares reductores obtenidos con la hidrólisis a base de $\mathrm{H}_{3} \mathrm{PO}_{4}$ con respecto al $\mathrm{H}_{2} \mathrm{SO}_{4}$ y $\mathrm{NaOH}$ en esta investigación.

La hidrólisis alcalina es capaz de separar tanto la hemicelulosa como la lignina sin tener grandes efectos sobre los demás componentes, como es la degradación de azúcares reductores (Malainine et al., 2003; Mussatto et al., 2006). Los reactivos más utilizados son $\mathrm{NaOH}, \mathrm{NH}_{3}$, $\mathrm{CaO}$ y $\mathrm{Ca}(\mathrm{OH})_{2}$, estos álcalis provocan la degradación del éster y cadenas laterales alterando la estructura de la lignina; de esta manera, se provoca la solvatación parcial de la hemicelulosa y una reducción en la cristalinidad de la celulosa; sin embargo, tienen la desventaja de que algunas bases como el $\mathrm{NaOH}$ (Sun y Cheng, 2002) y el KOH se convierten a sus sales, lo que podría ser una desventaja para su aplicación foliar directa o en soluciones nutritivas, como se observó en los resultados de la presente investigación. Desde este punto de vista, los tratamientos de hidrólisis 1 y 2 son más adecuados.

Los valores de Brix más altos fueron obtenidos con los

Cuadro 3. Rendimiento promedio del proceso de hidrólisis de las glóquidas de tuna de Opuntia albicarpa.

\begin{tabular}{|c|c|c|c|c|c|c|}
\hline $\begin{array}{l}\text { Tratamiento } \\
\text { No. }\end{array}$ & $\begin{array}{c}\text { Solución } \\
\text { (concentración, \%) }\end{array}$ & Baño María $\left({ }^{\circ} \mathrm{C}\right)$ & Degradación (\%) & $\begin{array}{c}\text { Azúcares } \\
\text { reductores }\left(\mathrm{mg} \mathrm{g}^{-1}\right) \\
\end{array}$ & Grados Brix & $\begin{array}{l}\text { Conductividad } \\
\text { eléctrica }\left(\mathrm{d} S \mathrm{~m}^{-1}\right)\end{array}$ \\
\hline 1.1 & \multirow{2}{*}{$\mathrm{H}_{2} \mathrm{SO}_{4}(15)$} & 60 & $67.9 a$ & $41.5 \mathrm{a}$ & $8.7 d$ & $0.88 d$ \\
\hline 1.2 & & $\sin$ & $49.8 a b$ & $21.6 \mathrm{~b}$ & $8.7 d$ & $0.86 \mathrm{de}$ \\
\hline 2.1 & \multirow{2}{*}{$\mathrm{H}_{3} \mathrm{PO}_{4}(15)$} & 60 & $27.4 \mathrm{bc}$ & $7.2 \mathrm{ef}$ & $17.1 \mathrm{a}$ & $1.24 \mathrm{c}$ \\
\hline 2.2 & & $\sin$ & $21.9 \mathrm{~cd}$ & $7.2 \mathrm{def}$ & $17.5 \mathrm{a}$ & $1.27 \mathrm{bc}$ \\
\hline 3.1 & \multirow{2}{*}{$\mathrm{NaOH}(15)$} & 60 & $46.8 \mathrm{abc}$ & 11.7 cde & $13.3 b$ & $1.51 \mathrm{a}$ \\
\hline 3.2 & & $\sin$ & $35.4 \mathrm{abc}$ & 9.9 cde & $13.0 \mathrm{~b}$ & $1.48 \mathrm{a}$ \\
\hline 4 & $\mathrm{H}_{2} \mathrm{O}_{2}(10)$ & $\sin$ & 14.7 ef & $1.4 \mathrm{~g}$ & $0.30 \mathrm{e}$ & $0.01 \mathrm{f}$ \\
\hline 5.1 & \multirow{2}{*}{$\mathrm{Ca}(\mathrm{OH})_{2}(0.2)$} & 60 & $11.3 \mathrm{fg}$ & $6.4 f$ & $0.43 \mathrm{e}$ & 0.03 ef \\
\hline 5.2 & & $\sin$ & $6.1 \mathrm{i}$ & 8.8 cde & $0.45 \mathrm{e}$ & 0.04 ef \\
\hline 6.1 & \multirow{2}{*}{$\mathrm{PHP}^{+}(10)$} & 40 & $1.8 j$ & $5.5 \mathrm{~g}$ & $10.9 \mathrm{bc}$ & 0.53 def \\
\hline 6.2 & & $\sin$ & $8.1 \mathrm{~g}$ & $5.2 \mathrm{~g}$ & $9.5 \mathrm{bc}$ & 0.67 def \\
\hline 7.1 & \multirow{2}{*}{$\mathrm{KOH}(15)$} & 60 & $7.4 \mathrm{~h}$ & $18.9 \mathrm{~cd}$ & $9.9 \mathrm{bc}$ & $1.40 \mathrm{a}$ \\
\hline 7.2 & & $\sin$ & $17.6 \mathrm{de}$ & $19.8 \mathrm{c}$ & $9.2 \mathrm{c}$ & $1.29 b$ \\
\hline 8 & $\mathrm{Ca}(\mathrm{OH})_{2}(10)$ & 60 & $2.9 \mathrm{i}$ & ND & $18.4 \mathrm{a}$ & $1.13 \mathrm{c}$ \\
\hline $\operatorname{Pr}>\mathrm{F}$ & & & $<0.01$ & $<0.01$ & $<0.01$ & $<0.01$ \\
\hline DSH (0.05) & & & 6.22 & 3.6 & 2 & 0.249 \\
\hline
\end{tabular}

Medias con letras iguales no son estadísticamente diferentes (Tukey, $\mathrm{P} \leq 0.05)$. DSH: diferencia significativa honesta, ND: no detectable. ${ }^{\dagger}$ La neutralización a pH 7 se consiguió con $\mathrm{KOH} 20 \%$ aplicando $12.2 \mathrm{~mL}$ para el tratamiento 6.1 y $16.1 \mathrm{~mL}$ para el tratamiento 6.2 . 
tratamientos de ácido fosfórico $\left(17.50{ }^{\circ} \mathrm{Bx}\right)$, seguidos del hidróxido de sodio $\left(13.3^{\circ} \mathrm{Bx}\right)$. Dichos valores bien pueden estar enmascarados por la presencia de sales, ya que por definición los ${ }^{\circ} \mathrm{Bx}$ miden sólidos solubles totales, por lo que es más confiable la cuantificación de azúcares reductores totales, dada su especificidad. En la presente investigación se observó que los valores más altos de ${ }^{\circ} \mathrm{Bx}$ se asociaron con la CE más alta. La Norma General del CODEX para zumos (jugos) y néctares de frutas (FAO/WHO, 2005) indica que las frutas de sandía, mango y uva deben tener por lo menos $8,13.5$ y $16^{\circ} \mathrm{Bx}$, respectivamente, por mencionar algunos ejemplos como referencia.

De acuerdo con Ulloa (2020; Com. Pers.)', la cantidad de glóquidas producidas en el período 2010 a 2015 en el municipio de Nopaltepec fue de 80.1 t, residuos orgánicos que no fueron aprovechados y que salieron del sistema de producción, convirtiéndose en una pérdida. Con base en los resultados de hidrólisis para el tratamiento más eficiente, para procesar $1 \mathrm{~kg}$ de glóquidas serían necesarios 30 $\mathrm{L}$ de la disolución con ácido sulfúrico (grado industrial); además, el material residual podría tener potencial para otros usos; por ejemplo, como sustrato para la producción de plántulas $u$ hongos, en tanto que el extracto por su contenido de minerales podría tener valor fertilizante. Este tipo de manejo podría dar valor agregado a un subproducto que se desecha.

\section{Contenido nutrimental de las glóquidas de tuna y de los extractos}

En el Cuadro 4 se presenta la concentración de nutrimentos de las glóquidas sin procesar y el contenido en los extractos de los procedimientos considerados como los más eficientes (solución con ácido sulfúrico, ácido fosfórico e hidróxido de potasio); es decir, que rindieron los valores mayores de degradación y liberaron mayor cantidad de azúcares reductores $\mathrm{y}^{\circ} \mathrm{Bx}$.

Con base en los reportes sobre la composición mineral de pulpa, semilla y cladodio del nopal tunero, en general, existe el siguiente orden de abundancia: $\mathrm{K}>\mathrm{Ca} \approx \mathrm{Mg}>\mathrm{P}>$ Mn, Fe, Zn y Cu (Díaz et al., 2007; El-Mostafa et al., 2014; Ochoa-Velasco y Guerrero-Beltrán, 2010). El presente estudio (Cuadro 4) indica que en las glóquidas los rangos de concentración (ppm) de K, Ca, Mg, Mn, Fe y Cu son mayores, en tanto que los de $\mathrm{P}$ y $\mathrm{Zn}$ fueron menores que los de otras partes del nopal tunero en su conjunto, encontrados por los autores antes indicados: K (2200552), Ca (590-162), Mg (984-88), Mn (3-2), Fe (94-1), Cu (3-0.4), P (1520-1.5) y Zn (14-1). De acuerdo con algunos investigadores (Ginestra et al., 2009; Loza-Cornejo y Terrazas, 2003), el Ca debe estar en forma de cristales de oxalato de calcio y el $\mathrm{Si}$ como cristales amorfos de $\mathrm{SiO}_{2}$ en los tejidos superficiales (epidermis e hipodermis) y pueden evitar que los tejidos subyacentes colapsen en tiempos de sequía al servir como envoltura protectora rígida. La presencia de taninos en las glóquidas, inferida por el color que adquirió el agua durante su hidratación, es otra característica de interés en la búsqueda de usos potenciales de dichas estructuras. De acuerdo con LozaCornejo y Terrazas (2003), los colorantes se alojan en el lumen de las células epidermales y protegen a las plantas contra parásitos u otros factores de estrés.

Cuadro 4. Contenido nutrimental promedio (ppm) de las glóquidas sin procesar y en el extracto, según el procedimiento de hidrólisis.

\begin{tabular}{ccccccccccccc}
\hline & $\mathrm{N}$ & $\mathrm{S}$ & $\mathrm{P}$ & $\mathrm{K}$ & $\mathrm{Ca}$ & $\mathrm{Mg}$ & $\mathrm{Fe}$ & $\mathrm{Cu}$ & $\mathrm{Zn}$ & $\mathrm{Mn}$ & $\mathrm{B}$ & $\mathrm{Si}$ \\
\hline \multirow{2}{*}{ Glóquidas } & 4550 & 140.0 & 200.0 & 3610 & 4970 & 1120 & 2171 & 12.2 & 27.1 & 122 & 50.0 & 224.5 \\
\multicolumn{7}{l}{ Contenido nutrimental de lo extractos de glóquidas hidrolizadas } & & & & & & \\
$\mathrm{H}_{2} \mathrm{SO}_{4}$ & 28.0 & 1833 & 53.3 & 2088 & 1355 & 312 & 113 & 10.7 & 17.5 & 42 & 0.8 & $\mathrm{nd}$ \\
& \pm 0.0 & \pm 77.3 & \pm 4.8 & \pm 86.3 & \pm 19.8 & \pm 0.4 & \pm 7.6 & \pm 0.1 & \pm 2.5 & \pm 4.3 & \pm 0.1 & \\
$\mathrm{H}_{3} \mathrm{PO}_{4}$ & 28.0 & 1750 & 57468 & 757 & 199 & 243 & 442 & 9.6 & 23.9 & $46 \pm 2.7$ & 9.3 & $\mathrm{nd}$ \\
& \pm 1.8 & \pm 41.7 & \pm 646 & \pm 4.7 & \pm 9.7 & \pm 8.8 & \pm 13.0 & \pm 0.4 & \pm 2.7 & & \pm 0.2 & \\
$\mathrm{KOH}$ & 23.3 & 1.47 & 22.5 & 751 & 422 & 283 & 491 & 14.9 & 17.1 & $42 \pm 2.9$ & 0.8 & $\mathrm{nd}$ \\
& \pm 2.0 & \pm 0.0 & \pm 2.4 & \pm 11.4 & \pm 18.5 & \pm 4.6 & \pm 26.5 & \pm 0.6 & \pm 1.4 & & \pm 0.1 &
\end{tabular}

Soluciones nutritivas comerciales ${ }^{\dagger+}$

\begin{tabular}{lccccccccccc} 
Hoagland- & 210 & 64 & 31 & 235 & 160 & 46 & 5 & & & & \\
Arnon & & & & & & & & & & & \\
Steiner & 167 & 67 & 31 & 277 & 183 & 49 & 3 & 0.02 & 0.11 & 1.97 & 0.44 \\
Wynd & 225 & $24-6$ & 137 & 112 & 167 & 25 & & & & & \\
\hline 'Las cifra precedida con el \pm después de los promedios $\left(\mathrm{n}=3\right.$ ) es la desviación estándar (Sd), ${ }^{++}$Hewitt (1966).
\end{tabular}


El conocimiento de elementos esenciales contenidos en las glóquidas, determinado en el presente estudio, proporciona las bases para investigar su uso como insumo en la nutrición de cultivos. Con este reciclado se podría contribuir a reducir el impacto ambiental negativo que se genera con este subproducto que se desecha en bolsas plásticas; asimismo, de forma innovadora se contribuye con conocimiento científico a la economía circular en el sector agrícola.

La hidrólisis mediante ácido sulfúrico generó los valores más altos de concentración de potasio y calcio, aunque este último fue menor que el determinado en las glóquidas sin hidrolizar, lo que indica la formación de compuestos insolubles. Con la solución de ácido fosfórico, los elementos más abundantes fueron el fósforo, seguido por el azufre y el hierro, lo cual implica la formación de compuestos insolubles entre el fósforo y el calcio. Finalmente, con el hidróxido de potasio se obtuvo mayor concentración de potasio, seguido de hierro y calcio. Si se descarta el enriquecimiento con el ion principal de la solución hidrolizante $(S, P$ y $K$ ), se observa que la hidrólisis con ácido sulfúrico solubilizó mayor cantidad de macronutrientes de las glóquidas.

Al comparar los resultados obtenidos con soluciones comerciales nutritivas se observó que la concentración de los macro y micronutrientes de las glóquidas hidrolizadas es mayor que en las soluciones de Hoagland-Arnon, Steiner y Wynd; en consecuencia, el subproducto del beneficio de la tuna tiene un gran potencial para su uso en fertirriego o en aspersiones foliares.

\section{Limitaciones e implicaciones del estudio}

La eficiencia de los procedimientos de hidrólisis que reporta la literatura para el aprovechamiento de la biomasa producida en los procesos agroindustriales con fines energéticos está determinada por la remoción de lignina, barrera para el acceso a los azúcares estructurales, y por la descomposición de la hemicelulosa para separar la celulosa; en general, dicha biomasa presenta mayor contenido de lignina (Morales et al., 2015; Com. Pers.), lo cual no sucede con las glóquidas, en las que hay sólo $5 \%$ de dicho compuesto; sin embargo, la mayor resistencia al ataque por los procedimientos ensayados, comparada con la de otros materiales lignocelulósicos, incluyendo la madera dura, en gran parte se debe a la celulosa embebidas en la matríz de a-L-arabinofuranosa (Vignon et al., 2004), lo que complica la eficiencia de los métodos para la extracción de los componentes

\footnotetext{
${ }^{5}$ Morales R. S. (2015)Hidrólisis ácida de celulosa y biomas lignocelulósica asistida con líquidos iónicos. Tesis de Doctorado. Universidad Autónoma de Madrid. Madrid, España. 248 p.
}

útiles. Los resultados obtenidos sugieren que en futuros estudios se ensayen pretratamientos de hidrólisis con soluciones diluidas ácidas/alcalinas, con el fin de eliminar la lignina, hacer más porosa la superficie de las glóquidas y aprovechar los derivados de la celulosa, hemicelulosa y otros componentes importantes como las oligosacarinas para su uso en la nutrición de cultivos. El subproducto o material no degradado podría tener uso como sustrato en viverismo.

Con el presente estudio se logró determinar el contenido de elementos esenciales de las glóquidas hidrolizadas y compararlo con la composición natural de estas estructuras; sin embargo, es necesario realizar pruebas en las cuales la solución obtenida por hidrólisis se aplique a cultivos para determinar en campo su potencial como fertilizante. El conocimiento sobre la estructura externa y composición de las estructuras estudiadas también permitió visualizar su potencial de uso como sustrato en la producción de hongo Pleurotus sp., cuya investigación está en curso.

\section{CONCLUSIONES}

Las glóquidas de la tuna tienen barbas retrorsas y sus dimensiones son comparables a las glóquidas de otras especies de Opuntia. El 83.3 \% de la pared celular está compuesta por celulosa y hemicelulosa en proporciones similares. Sólo el 5.3 \% de ella es lignina. Los mayores porcentajes de degradación y obtención de azúcares reductores totales se obtuvieron a partir de la hidrólisis con ácido sulfúrico, ácido fosfórico e hidróxido de potasio. Los tratamientos de hidrólisis que rindieron los valores mayores de degradación, azúcares reductores $\mathrm{y}^{\circ} \mathrm{Bx}$ fueron los basados en $\mathrm{H}_{2} \mathrm{SO}_{4}, \mathrm{KOH}$ y $\mathrm{H}_{3} \mathrm{PO}_{4}$; de éstos, la hidrólisis con ácido sulfúrico produjo la mayor cantidad de azúcares reductores y minerales. La presente investigación evidencia la posibilidad de utilizar los extractos de las glóquidas para usos agronómicos en la nutrición de cultivos.

\section{AGRADECIMIENTOS}

El posgrado del Departamento de Suelos y los autores agradecen la beca otorgada por el CONACYT y Universidad Autónoma Chapingo (UACh) al primer autor para llevar a cabo sus estudios y la presente investigación; asimismo, se extiende reconocimiento al LANISAF de la UACh y a la UME del Colegio de Postgraduados, así como a su personal técnico por el servicio brindado en microscopía electrónica de barrido. 


\section{BIBLIOGRAFÍA}

Aguilar Z. A., J. Jolalpa B. y D. Garza G. (2008) Guía para cultivar nopal tunero en el estado de Hidalgo. Folleto para Productores No. 15. Campo Experimental Pachuca, Instituto Nacional de Investigaciones Forestales, Agrícolas y Pecuarias. Pachuca Hidalgo. 35 p.

Alcántar G. G. y M. Sandoval V. (1999) Manual de Análisis Químico de Tejido Vegetal: Guía de Muestreo, Preparación, Análisis e Interpretación. Sociedad Mexicana de la Ciencia del Suelo. Chapingo, Estado de México. 155 p.

Callejas-Juárez N., J. Matus-Gardea, J. A. García-Salazar, M. A. MartínezDamián y J. M. Salas-González (2009) Situación actual y perspectivas de mercado para la tuna, el nopalito y derivados en el Estado de México, 2006. Agrociencia 43:73-82.

Chahdoura H., P. Morales, J. Barreira, L. Barros, V. Fernández-Ruiz, L. Ferreira and L. Achour (2015) Dietary fiber, mineral elements profile and macronutrients composition in different edible parts of Opuntia microdasys (Lehm.) Pfeiff and Opuntia macrorhiza (Engelm.). Food Science and Technology 64:446-451, https:// doi.org/10.1016/j.Iwt.2015.05.011

Díaz M. E. M., E. M. Rodríguez R. and C. Díaz R. (2007) Chemical characterization of Opuntia dillenii and Opuntia ficus indica fruits. Food Chemistry 103:38-45, https://doi.org/10.1016/j. foodchem.2006.06.064

Dieter R. A., L. R. Whitehouse and R. Gulliver (2017) Cactus spine wounds: a case report and short review of the literature. Wounds 29:E18-E21.

Doctoroff A., A. T. Vidimos and J. S. Taylor (2000) Cactus skin injuries. Cutis 65:290-292.

Domínguez-Domínguez M. M., A. Álvarez-Castillo, M. Granados-Baeza y F. Hernández-Campos (2012) Estudio de la cinética del pre tratamiento e hidrólisis ácida del bagazo de caña de azúcar Revista Iberoamericana de Polímeros 13:200-211.

El-Mostafa K., Y. El Kharrassi, A. Badreddine, P. Andreoletti, J. Vamecq, M. S. El Kebbaj, ... and M. Cherkaoui-Malki (2014) Nopal cactus (Opuntia ficus-indica) as a source of bioactive compounds for nutrition, health and disease. Molecules 19:14879-14901, https://doi. org/10.3390/molecules 190914879

FAO/WHO, Food and Agriculture Organization/World Health Organization (2005) Norma general del CODEX para zumos (jugos) y néctares de frutas. CODEX STAN 247. FAO/WHO Food Standards. Rome. 21 p, http://www.fao.org/input/download/standards/10154/ CXS_247s.pdf (May 2021).

Ford A. M., S. T. Haywood and D. R. Gallo (2019) Novel method for removing embedded cactus spines in the emergency department. Case Reports in Emergency Medicine 2019:6062531, https://doi org/10.1155/2019/6062531

Gindl-Altmutter W. and J. Keckes (2012) The structure and mechanical properties of spines from the cactus. Bioresources 7:12321237.

Ginestra G., M. L. Parker, R. N. Bennett, J. Robertson, G. Mandalari, A. Narbad and K. W. Waldron (2009) Anatomical, chemical, and biochemical characterization of cladodes from prickly pear [Opuntia ficusindica (L.) Mill.]. Journal of Agricultural and Food Chemistry 57:10323-10330, https://doi.org/10.1021/jf9022096

Hewitt E. (1966) Sand and Water Culture Methods Used in the Study of Plant Nutrition. 2nd edition. Commonwealth Agricultural Bureaux. Farnham Royal, England. 547 p.

Kalegowda P., D. J. Haware, S. Rajarathnam and M. N. Shashirekha (2015) Minerals of cactus (Opuntia dillenii): cladode and fruit. Current
Science 109:2295-2298, https://doi.org/10.18520/v109/ i1 2/2295-2298

Loza-Cornejo S. and T. Terrazas (2003) Epidermal and hypodermal characteristics in North American Cactoideae (Cactaceae) Journal of Plant Research 116:27-35. https://doi.org/10.1007/ s10265-002-0066-2

Malainine M. E. , A. Dufresne, D. Dupeyre, M. Mahrouz, R. Vuong and M. R. Vignon (2003) Structure and morphology of cladodes and spines of Opuntia ficus-indica. Cellulose extraction and characterisation. Carbohydrate Polymers 51:77-83, https://doi. org/10.1016/S0144-8617(02)00157-1

Martínez-González C., C. Gallegos-Vázquez, I. Luna-Vega y R. GarcíaSandoval (2015) Opuntia leiascheivariana, una nueva especie de cactacea del Estado de Hidalgo, México. Botanical Sciences 5:517-529, https://doi.org/10.17129/botsci.247

Monsalve G. J. F., V. I. Medina P. y A. A. Ruiz C. (2006) Producción de etanol a partir de la cáscara de banano y de almidón de yuca. Dyna 73:21-27.

Mussatto S. I., G. Dragone, M. Fernández, G. J. M. Rocha y R. Inés (2006) Efecto de los tratamientos de hidrólisis ácida e hidrólisis alcalina en la estructura del bagazo de malta para liberación de fibras de celulosa. XXII Interamerican Congress of Chemical Engineering. Interamerican Confederation of Chemical Engineering. Asociación Argentina de Ingenieros Químicos. Buenos Aires, Argentina. $10 \mathrm{p}$.

Mustafa-Odat T. A., M. Al-Tawara-Jebreel and E. Hussein-Hammouri (2014) Prickly pear spine keratoconjunctivitis. Middle East African Journal of Ophthalmology 21:61-65, https://doi org/10.4103/0974-9233.124100

Naranjo C. D., L. Alamilla-Beltrán, G. F. Gutiérrez-López, E. Terres-Rojas y J. Solorza-Feria, S. Romero-Vargas, ... y R. Mora-Escobedo (2016) Aislamiento y caracterización de celulosas obtenidas de fibras de Agave salmiana aplicando dos métodos de extracción ácido-alcali. Revista Mexicana de Ciencias Agrícolas 7:31-43, https://doi.org/10.29312/remexca.v7i1.368

Ochoa-Velasco C. y J. Guerrero-Beltrán (2010) La tuna: una perspectiva de su producción, propiedades y métodos de conservación. Temas Selectos de Ingeniería de Alimentos 4:49-63.

Reis L. H. O., A. O. Rodrigues, S. I. Mussatto, A. R. Gonçalves y G. J. M. Rocha (2007) Hidrólisis enzimática de la celulosa obtenida a partir del pseudotronco de la bananera pretratada con solución de ácido sulfúrico. Depto. de Biotecnología, Universidad de São Paulo, São Paulo, Brasil. 9 p.

Scheinvar L., G. Olalde-Parra and C. Gallegos-Vázquez (2015) A new species of the genus Opuntia (Cactaceae) for the state of Veracruz, Mexico. Botanical Sciences 93:33-39, https://doi. org/10.17129/botsci.133

Sun Y. and J. Cheng (2002) Hydrolysis of lignocellulosic materials for ethanol production: a review. Bioresource Technology 83:1-11, https://doi.org/10.1016/S0960-8524(01)00212-7

Van Soest P., J. Robertson and B. Lewis (1991) Methods for dietary fiber, neutral detergent fiber, and nonstarch polysaccharides in relation to animal nutrition. Journal of Dairy Science 74:35833597, https://doi.org/10.3168/jds.S0022-0302(91)78551-2

Vignon M R, L. Heux, M. E. Malainine and M. Mahrouz (2004) Arabinancellulose composite in Opuntia ficus-indica prickly pear spines. Carbohydrate Research 339:123-131, https://doi.org/10.1016/j. carres.2003.09.023

Yousef K. A., A. J. Goshinska, Z. P. Bailowitz and R. Amini (2017) Cactus thorn detection using bedside ultrasound. Internal and Emergency Medicine 12:545-546, https://doi.org/10.1007/s11739-0161515-6 
